\title{
Exploring the Role of Attitudinal vs. Situational Ambivalence in Consumer Choice: An Abstract
}

\author{
Piyush Sharma, Cheryl Leo, Anish Nagpal, and Yuwei Jiang
}

\begin{abstract}
Ambivalence reflects the coexistence of positive and negative evaluations of an attitude object, and there is mixed evidence about its impact on consumer decision-making and behavior, with some studies showing ambivalent attitudes as weak and less predictive of behavior, whereas others find them to be more predictive of behavioral intentions. Prior research also does not empirically examine the relationships among ambivalence, indifference, and dissonance despite their similarities and differences with each other. We address these gaps with a conceptual framework based on an extensive review of consumer ambivalence literature and two experimental studies that explore the role of consumer ambivalence in consumer choice. Overall, our findings show that attitudinal and situational ambivalence do vary in their effects on consumer choice between hedonic and utilitarian options. We also show that consumer dissonance and indifference play important roles in this process. These findings extend current research on consumer ambivalence as well as on consumer choice by distinguishing between the roles of the two types of ambivalence and the roles of consumer dissonance and indifference on the choice between hedonic and utilitarian options. Besides these conceptual contributions, our findings also have important managerial implications for marketers of hedonic versus utilitarian products and services.
\end{abstract}

\footnotetext{
P. Sharma $(\varangle)$

Curtin University, Bentley, Australia

e-mail: piyush.sharma@curtin.edu.au

C. Leo

Murdoch University, Murdoch, Australia

e-mail: c.leo@murdoch.edu.au
}

\author{
A. Nagpal \\ University of Melbourne, Melbourne, Australia \\ e-mail: anagpal@unimelb.edu.au \\ Y. Jiang \\ Hong Kong Polytechnic University, Hong Kong SAR \\ e-mail: yuwei.jiang@polyu.edu.hk
}

